\title{
Ecritures de soi, écritures des limites
}

Escrituras de si, escrituras de limite

Writings of one self, writings of limits

\section{Jean-François Chiantaretto ${ }^{1}$}

Université Paris 13, Paris, França

\section{Résumé}

Les pathologies des limites correspondent à un surinvestissement des frontières de la psyché, du fait des atteintes précoces portées à l'intégrité de son territoire. Cette problématique est éclairée par les écritures de soi, avec lesquelles le récit présente une mise en scène de l'interlocution interne, de la représentation de soi dans le regard de l'autre. Le rapprochement est d'autant plus éclairant lorsque l'écriture de soi vient témoigner d'une expérience traumatique caractérisée par la liaison de la menace vitale et de la menace du sentiment d'appartenance humaine, de la disparition de soi et de la disparition à soi. L'œuvre d'Imre Kertész sera étudiée dans cette perspective.

Mots-clés: Interlocution interne, narcissisme, sentiment d'appartenance humaine, sentiment d'exister, témoin survivant, trauma.

\section{Resumo}

As patologias dos limites correspondem a um superinvestimento das fronteiras da psique devido aos abalos precoces dirigidos à integridade de seu território. Esta problemática é esclarecida pelas escritas de si nas quais a narrativa apresenta uma encenação da interlocução interna, da representação de si diante do olhar do outro. Essa aproximação é ainda mais esclarecedora quando a escrita de si vem testemunhar uma experiência traumática caracterizada pela ligação entre a ameaça à vida e a ameaça ao sentimento de pertença humana, a disaparição de si e a disaparição em si. A obra de Imre Kertesz será estudada nesta perspectiva.

Palavras-chave: interlocução interna, narcisismo, sentimento de pertença humana, sentimento de existir, testemunho do sobrevivente, trauma. 


\begin{abstract}
The pathology of limits correspond to a super-investment of the boundaries of the psyche, due to the early damages to the integrity of its territory. This question is clarified by ones experience, with which the report presents a scene of internal exchange, on the representations of ones self on another's point of view. The approximation is even more clarifying when the experience of one's self are a witness of a traumatic experience characterized by the conection between the threat to life and the threat to the feeling of belonging, and between loosing one's self and disappearing before one's self. The work of Imre Kertész will be studied unde this perspective.
\end{abstract}

Keywords: Internal exchange, Narcisism, Feeling of human belonging, Feeling of living, Witness of survivor, Trauma.

Et si la question de l'écriture de soi aidait l'analyste à penser la fonction particulière des limites que s'imposent certains patients dans l'investigation de leur psychisme? Ces limites renverraient ${ }^{2}$ aux limites qui manquent au sujet pour se sentir exister, au sens où quelqu'un $a$ manqué pour différencier le moi du nonmoi, ce qui est à moi et ce qui n'est pas à moi. La question de l'être est devenue de ce fait une question de territoire, de propriété : non plus qui suis-je?, mais où suis-je ? ou est-ce à moi ou à l'autre? Les limites de l'investigation viendraient ainsi tout à fois donner une forme, fut-elle non adaptée, à la souffrance vide (sans contenant) attachée au sentiment d'exister dans un lieu mutilé et exprimer l'orgueil vide (sans contenu), attaché à la revendication de ce lieu mutilé comme seul lieu possible de l'être. Tel est du moins le parti pris : chercher dans les écritures de soi de quoi mieux penser les pathologies des limites et plus largement, la question des limites en psychopathologie. Les pathologies des limites, c'est-à-dire un ensemble très divers de figures psychopathologiques du narcissisme, ne relevant en propre ni de la névrose, ni de la psychose - le narcissisme: la représentation de soi dans le regard de l'autre.

Ces pathologies, qui occupent de plus en plus le devant de la scène clinique, ont pour trait commun l'hypothèque frappant la possibilité de se parler en parlant, l'expérience de l'intériorité dans la parole, c'est-à-dire les sources émotionnelles et affectives du langage. Le défaut de confiance dans les mots en est la manifestation la plus nette. 
La parole suppose la confiance dans les mots, qui elle-même repose sur l'expérience du je dans la parole. A la racine de toute parole adressée, il y a la possibilité de se parler. Avoir (suffisamment, mais pas trop) confiance dans les mots pour parler et dire je en s'adressant à autrui, suppose d'avoir été suffisamment bien parlé par l'autre dans la situation de dépendance vitale des premiers temps de la vie - suffisamment bien pour pouvoir se parler à soi-même en présence de l'autre.

Sans doute commence-t-on à penser « pour de vrai » à partir du moment où l'on se sent abandonné par ses certitudes premières, celles-là mêmes qui semblaient indissociables de soi. Lorsque j'ai commencé à m'intéresser à ce que j'ai ensuite nommé les écritures de soi, en empruntant le terme à Michel Foucault, j'avais la conviction que c'était là un investissement de l'auto-présentation, de la représentation de soi, pour l'essentiel à la fois hétérogène et rival à ce qui pouvait s'expérimenter dans l'espace analytique. M'autorisant des travaux de Philippe Lejeune $^{3}$, qui permettaient de dépasser la définition de l'autobiographie en termes de genre littéraire, j'avais alors tendance à penser toute écriture de soi sur le modèle autobiographique, avec l'idée de la mise en acte d'un fantasme d'auto-engendrement dans la représentation de soi en train de se présenter au regard de l'autre ${ }^{4}$. L'écriture de soi était ainsi supposée dans tous les cas présenter une attestation d'identité, à l'opposé donc de la visée analytique de déconstruction/reconstruction du tissage des identifications. L'autobiographe et sa revendication d'une unité indivisible, en me servant de modèle et d'emblème pour les écritures de soi, m'amenait ainsi à les penser trop exclusivement et trop indistinctement en termes de résistance. Une résistance à la mise en œuvre des identifications multiples soutenant l'identité plurielle propre à tout sujet, soit une résistance au devenir caractérisant le travail psychique dans l'analyse : voilà qui je suis $v s$ voilà qui je deviens.

Mon point de vue s'est considérablement assoupli au fil des années. D'abord, j'ai pu mesurer, avec les journaux intimes et leurs dérivés, combien le travail psychique $\mathrm{du}$ diariste se rapprochait de ce qu'il faut souhaiter à tout analysant concernant l'acceptation d'un soi pluriel et relationnel. Surtout, j'ai pu progressivement prendre en compte, qu'audelà de la différenciation des différents types d'écriture de soi et en particulier, de la différenciation autobiographie/journal intime, tout texte relevant de l'écriture de soi, quel que soit le genre et quel que soit l'auteur, mettait en scène un conflit entre deux positions psychiques : d'une part, la présentation de soi au sens d'une 
attestation d'identité, d'autre part, le témoignage d'une altération de soi et d'une altération en soi ${ }^{5}$.

Mais l'essentiel dans ce changement de perspectives est venu des analysants, qui m'ont appris que l'écriture de soi menée parallèlement n'était pas toujours une résistance à la cure - à la cure comme processus d'auto-transformation lié à l'expérience de soi en tant qu'elle est garantie par l'écoute de l'autre. Une telle écriture de soi peut aussi constituer un prolongement créatif de ce processus, ou même encore une condition de possibilité, au sens d'une résistance à la résistance du patient et/ou de l'analyste. Dans certains cas difficiles, relevant de pathologies des limites, l'écriture de soi peut apparaître comme indispensable pour s'éprouver en train d'être en présence de l'autre, ce qui constitue à la fois le coeur et l'outil de la visée analytique. Il s'agit alors d'histoires marquées par des expériences traumatiques précoces, avant l'accès au $\mathrm{Je}^{6}$, c'est-à-dire des traumas négatifs, dans une perspective bien explicitée par André Green : quelque chose n'a pas eu lieu, au sens où ce qui a eu lieu n'a pas eu de lieu psychique pour s'inscrire. L'effraction traumatique procède non pas d'un trop mais d'un vide (d'un évidement), en deçà de toute expérience accessible après coup par la levée du refoulement.
Il m'est ainsi apparu, dans l'aprèscoup, que mon intérêt pour les écritures de soi procédait pour une bonne part des difficultés cliniques rencontrées avec des patients ayant subi un trauma précoce, dont la caractéristique était l'impossibilité à s'inscrire psychiquement, aucun élément de l'expérience ne pouvant accéder à la représentation. Chez ces patients, l'expérience traumatique s'est inscrite négativement, dans l'impossibilité même de s'inscrire. Elle est présente au titre d'une menace non représentable, activée par le cours même du fonctionnement psychique et non par un élément psychique déclencheur, qui serait isolable. Comme si était atteinte la possibilité pour la psyché de se représenter elle-même dans l'activité de représentation, fragilisant ainsi toute représentation.

Dans cette perspective ouverte, après Ferenczi, par Winnicott, lorsqu'il avance l'idée d'une crainte de l'effondrement, l'expérience traumatique ne trouve inscription, au moins une inscription potentielle, que dans le travail psychique de l'autre. L'analyste est amené à ressentir pour le patient la menace attachée à tout affect et toute émotion, à la ressentir dans le transfert d'un vide exigeant d'être accueilli et traduit dans sa propre psyché, comme une nécessité vitale d'interroger les sources émotionnelles et 
affectives de son propre fonctionnement psychique.

Avec ce type de cas, l'écriture de soi parallèlement à la cure pourrait être envisagée comme la tentative de construire un espace contenant intermédiaire, n'appartenant ni seulement au patient, ni seulement à l'analyste, permettant un travail de co-pensée impossible autrement. En considérant l'écriture de soi comme objet de recherche psychanalytique, en l'approchant comme un dispositif par lequel l'auteur se voit ou se donne à voir dans le regard supposé du lecteur, il s'agit de penser le vide menaçant l'infans ${ }^{7}$ mal accueilli et mal traduit par la psyché maternelle. Les patients-limites en témoignent au présent, dès lors qu'ils prennent le risque de s'adresser à un analyste. Lequel analyste aura la lourde charge d'accueillir ce vide en lui au titre d'une empreinte des toutes premières relations du patient, ce qui ne sera possible qu'à condition de pouvoir utiliser cette empreinte doublement étrangère pour interroger les modalités relationnelles et émotionnelles façonnage de sa propre psyché.

En mettant en scène avec des mots la manière dont l'auteur se voit vu, les écritures de soi nous montrent en marche ce qui ne marche pas, ou mal, dans les pathologies des limites : la possibilité de se parler, c'est-à-dire d'une expérience intérieure de soi par les mots. Se parler suppose une confiance minimale dans les mots, un rapport suffisamment confiant au langage à la fois comme sol commun avec les autres et comme promesse d'une écoute. Cette confiance est conditionnée dans les premiers temps de la vie par la fiabilité de la psyché maternelle, par sa capacité à donner sens aux ressentis et aux besoins du nourrisson en s'y adaptant et en les traduisant en termes de désir - ce qui précisément fait défaut dans les pathologies des limites.

La défaillance de l'autre comme garant d'une écoute caractérise aussi l'expérience traumatique des témoins survivants d'une catastrophe collective. Lorsque j'en suis venu à étudier ce type de témoignages, tout particulièrement avec la Shoah, je me suis longtemps refusé à approfondir le rapprochement entre la désolation, l'abandon et la défection du prochain vécue par les déportés dans les camps nazis, et la désolation, l'abandon et la défection du prochain inscrite par la défaillance psychique de l'environnement du nourrisson. Cela tenait sans doute à une méfiance, justifiée, vis-à-vis de toute dérive psychologisante, fut-elle à tonalité psychanalytique, mais cette méfiance, certes légitime eu égard aux enjeux et à leur complexité, manifestait aussi un trouble. J'ai eu bien du mal à pleinement admettre que dans ces investigations autour 
des témoignages de survivants, j'étais mu par un questionnement concernant la possibilité, en étayage sur la psyché de l'autre, de mettre en mots ce type de traumas précoces - traumas précoces affectant donc, en deçà de toute représentation, le façonnage émotionnel et relationnel de la psyché.

Pour le dire plus simplement, en m'inspirant d'une formulation de Louise Grenier dans un livre récent, c'est toute « la question du rapport entre les récits des survivants des camps de la mort et l'absence de récit de ceux qui ont traversé des agonies primitives $»^{8}$. En d'autres termes, c'est la question de savoir en quoi les témoins survivants nous disent quelque chose de la possibilité d'une défaillance mortelle de l'environnement, plus ou moins présente pour chacun d'entre nous au début de la vie, et aussi en quoi ils nous disent quelque chose du besoin vital de la psyché de l'autre pour exister, du besoin vital d'un témoin pour écrire sa vie - étant bien entendu que le témoignage des survivants a sa nécessité intrinsèque, tant du point de vue du survivant, de son groupe d'appartenance que du point de vue de l'ensemble humain'.

À partir de la résistance intérieure du survivant menacé d'anéantissement par l'autre, il s'agit de penser l'intériorité comme lieu de résistance face à la menace d'une disparition de l'autre, inscrivant la possible disparition de soi. Et d'ouvrir la question posée par les pathologies des limites : comment trouver en soi la force d'exister lorsque le soi reste à trouver?

L'enjeu reste néanmoins le même pour tous : la vie n'est vivable qu'en étant racontable, c'est-à-dire écrite potentiellement pour quelqu'un. Tout récit suppose un destinataire, tout récit constitue une scène d'interlocution : il s'agit de parler à quelqu'un. Dans l'écriture de soi, il s'agit plus spécifiquement de mettre en scène un dialogue intérieur, la scène intérieure requérant le lecteur comme témoin garant d'une écoute. Qu'advient-il de cette visée lorsque cette scène intérieure est attaquée par une expérience traumatique mettant en cause l'appartenance humaine, lorsqu'est attaquée la possibilité d'une parole fondée sur le dialogue intérieur avec les autres en soi, c'est-à-dire la confiance dans les mots pour se dire? Dans les récits des témoins survivants de la Shoah, l'attaque de la subjectivité est devenue la matière même du témoignage. Le témoignage est alors condamné à affronter la menace d'un redoublement du trauma dans la réduction de l'écriture de soi à la production d'une preuve, vérifiable par les historiens.

Après celle de Primo Levi ${ }^{10}$, l'œuvre d'Imre Kertész est ici extraordinairement précieuse, précisément parce que l'écriture de soi y met en scène 
le dialogue intérieur du témoin, le lien indéfectible entre le besoin de se parler et le besoin de l'autre pour s'entendre. Et le met en scène à la fois tel qu'il est éprouvé, dans tous les sens du terme, chez les témoins survivants et tel qu'il peut l'être par chacun d'entre nous.

\section{Raconter pour vivre ou vivre pour raconter?}

Comme Primo Levi, Imre Kertész est né à l'écriture après Auschwitz, et à partir d'Auschwitz. Chez l'un et l'autre, l'écriture assume sa dimension subjective, intègre un projet testimonial et le déborde. Mais en deçà même de leur style, très opposé, leur différence porte sur le statut de l'altération de l'écriture par l'expérience traumatique, voire sur la nature même de l'altération traumatique dans l'écriture.

Chez Primo Levi, la séparation est nette entre les textes testimoniaux consacrés à Auschwitz et les autres, nouvelles ou essais, même si on retrouve indéniablement une unité du style narratif, marqué par le primat de l'observation et de la description. L'altération traumatique de la subjectivité est, au moins officiellement, maintenue dans les textes consacrés à Auschwitz et tout l'effort de l'écriture semble être effectivement de l'observer et de la décrire comme de l'extérieur. Même l'altération du témoignage par le témoignage lui-même, en tant qu'il sécrète une «mémoire artificielle», devenue une préoccupation de plus en plus envahissante après la seconde édition de Si c'est un homme, reste de l'ordre de l'observation. Comme si Primo Levi cherchait à maintenir, de plus en plus difficilement, la possibilité d'un salut dans l'écriture, ou plutôt, d'un salut amené par l'écriture. Chez lui, l'écriture est née de la survie et participe à la survie : le besoin de raconter est donné pour salvateur. Et lorsqu'il proclame «j'écris parce que je suis chimiste $»^{11}$, il s'agit de rendre grâce à sa formation de chimiste, qui l'a probablement sauvé à Auschwitz Elle lui a permis non seulement d'être affecté la journée à une tâche plus supportable, mais surtout de maintenir en lui une position d'observateur, rendant possible de vivre l'expérience traumatique comme une expérimentation catastrophique d'une zone intermédiaire, de l'inhumain dans l'humain - c'est d'ailleurs tout le sens du titre, $S i$ c'est un homme.

Chez Imre Kertész, au contraire, la séparation n'est pas nette entre les textes consacrés à Auschwitz et les autres, et l'entreprise testimoniale est en fait déplacée, voire en un sens généralisée à $1^{\prime}$ ensemble ${ }^{12}$. Etre sans destin relate bien l'arrestation, puis la déportation à Auschwitz et le transfert à Buchenwald, enfin la libération et le retour. Mais, d'une 
part, l'expérience des camps nazis littéralement occupe les autres textes, et d'autre part, Etre sans destin, comme Kaddish pour l'enfant qui ne naîtra pas et jusqu'à un certain point Un autre, entend témoigner avant tout de l'expérience intérieure d'un sujet confronté à l'attaque traumatique de la possibilité de raconter sa vie, à défaut de pouvoir la vivre. Et contrairement à Levi, le témoignage associe Auschwitz à l'avant-Auschwitz (l'enfance) et à l'après-Auschwitz (la vie dans la Hongrie communiste). Jusqu'au présent de l'écriture, il s'agit sans cesse de vivre une «existence incroyable », l'incroyable d'exister. L'écriture vient matérialiser la continuité d'une expérience de soi comme doté d'une vie invivable, où la seule issue est de «faire de sa vie une réalité racontable ».

Existe-t-il un moyen de comprendre qui ne soit pas une possession, une prise en son pouvoir de ce que l'on comprend? Par exemple, se perdre dans un récit et là tomber dans un piège sans issue...

Ma vie est-elle autre chose qu'un tel récit ? Un seul moyen: en faire une réalité racontable $[\ldots]$ Ce récit raconterait le combat incessant, imperceptible, pareil aux métamorphoses de l'embryon, qui a autrefois commencé en moi afin que je remonte à la surface de la conscience depuis les profondeurs insondables de l'existence, puis que je fasse accepter cette existence (la mienne) par cette conscience toute nouvelle. ${ }^{13}$

Parler sa vie à quelqu'un: telle serait la seule façon de rendre sa vie vivable, au double sens de la supporter et de poursuivre le processus d'incarnation mis en œuvre pendant la gestation. Chez Primo Levi, la douleur dans le récit d'Auschwitz était liée à la réactivation de l'expérience traumatique, mais surtout à l'incapacité de l'écriture testimoniale, progressivement avérée, de consacrer et légitimer rétroactivement l'effort de vivre pour raconter - ce qui encore une fois aurait supposé la reconnaissance définitive et irréductible d'Auschwitz, son intégration inconditionnelle dans l'écriture de l'histoire des hommes. Avec Imre Kertész, c'est toute l'écriture, et pas seulement le récit d'Auschwitz, qui porte la douleur: elle n'est pas donnée comme seulement liée à Auschwitz, ni aux insuffisances traumatiques de la réception du témoignage, qui fragilisent après coup l'effort de survivre pour raconter.

L'enjeu n'est plus de vivre pour raconter Auschwitz, de ne pas trahir dans sa vie le «désir primordial et violent de raconter » à la racine de la survie. Il s'agit plutôt de raconter pour vivre, de rendre racontable sa vie, avant, pendant et après Auschwitz, dans la continuité d'un étonnement d'être, d'une expérience 
réitérée de l'incroyable de la vie, détachée de la mort, hors la peur et l'oubli de la mort.

Dans la mesure où Auschwitz oblige à vivre dans le présent hors la loi du meurtre déshumanisé et désindividualisé, l'expérience de l'incroyable est radicalement renforcée. Le récit proposé par Être sans destin, même s'il présente minutieusement la réalité quotidienne d'Auschwitz, nous montre un Imre Kertész $\mathrm{y}$ ayant connu, du moins à certains moments, le plein sentiment d'exister, jusqu'à évoquer, certes dans l'après-coup, des instants de bonheur :

[...] là-bas aussi, parmi les cheminées, dans les intervalles de la souffrance, il y avait quelque chose qui ressemblait au bonheur. Tout le monde me pose des questions à propos des vicissitudes, des «horreurs »: pourtant en ce qui me concerne, c'est peut-être ce sentiment-là qui restera le plus mémorable. Oui, c'est de cela, du bonheur des camps de concentration, que je devrais parler la prochaine fois, quand on me posera des questions. Si jamais on m'en pose. Et si je ne l'ai pas moi-même oublié. ${ }^{14}$

Il reste que l'étonnement d'être se mêle à l'ennui, lié à l'impossibilité d'être :

[...] même à Auschwitz, on pouvait s'ennuyer - à condition d'être un privilégié. Nous attendions - à bien $\mathrm{y}$ réfléchir, nous attendions que rien ne se passe. Cet ennui, avec cette étrange attente : je crois que c'est cette impressionlà, à peu près, oui, qui en réalité caractérise vraiment Auschwitz- à mes yeux en tout cas. $^{15}$

Tous les livres de Kertész proclament une continuité de l'expérience de soi avant, pendant et après Auschwitz, qui ne doit donc pas être confondue avec l'expérience catastrophique elle-même d'ailleurs, la continuité n'existe peut-être qu'à être proclamée par l'écriture. Il reste que la proclamation doit être reçue comme elle se donne: du point de vue de l'expérience de soi, Auschwitz ne fait que radicaliser à l'extrême la certitude intérieure d'être de plain pied, depuis toujours, avec un environnement défaillant.

Là où l'écriture testimoniale ne protège pas Primo Levi de la défaillance de l'autre réactivée par le témoignage, là où il a le sentiment cruel dans Naufragés et rescapés d'avoir échoué à participer à l'écriture de l'histoire et à contribuer au «plus jamais ça », il nous aide par sa douleur même à affronter Auschwitz. Au risque peut-être de nous aider à nous décharger d'Auschwitz. En effet, sans doute peut-on utiliser Primo Levi, plus facilement qu'Imre Kertész, pour en rester au devoir de mémoire, certes nécessaire, et prendre le risque d'oublier la présence d'Auschwitz. La présence d'Auschwitz: 
non pas seulement au sens du déni et de la menace qui continuent de peser sur les Juifs, non pas seulement au sens de la perpétration d'autres génocides après la Shoah, mais aussi au sens de l'entrée avec Auschwitz - puis Hiroshima - dans l'ère d'une possible destruction de l'humain, prenant la forme inédite d'un Tout est possible, attesté par la prolifération des techno-sciences dans tous les secteurs de la vie et de la pensée, par-delà divers supports idéologiques, politiques ou religieux.

Avec Imre Kertész, contrairement à Primo Levi, l'écriture testimoniale se fond dans l'écriture de soi - à moins que ce ne soit l'inverse - pour s'installer au présent dans la catastrophe et permettre à l'auteur de survivre à sa survie.

\section{« Faire de sa vie une réalité racontable »}

Tous les ouvrages de Kertész mettent ainsi en scène un dialogue intérieur constamment menacé de virer au soliloque. Ce dialogue intérieur est donné comme le seul lieu possible d'habitation psychique, le seul mode possible d'expérience de soi, d'un soi voué à la plus extrême précarité. Mais le dialogue intérieur vient ici manifester un dialogue empêché avec les autres, pris à témoin de cet empêchement étant entendu que ce dialogue intérieur et la sollicitation de l'autre comme témoin plus ou moins empêché fonctionnent très différemment dans l'œuvre de Kertész, selon qu'il s'agisse d'un texte plutôt autobiographique ou plutôt fictionnel étant entendu que cette différenciation est chez lui constamment mise à mal.

Comme d'autres survivants, Imre Kertész est né à l'écriture après Auschwitz, et à partir d'Auschwitz. Chez lui, comme chez d'autres écrivains de la catastrophe, l'écriture assume sa dimension subjective, intègre un projet testimonial et le déborde. Mais la singularité de Kertész réside dans le destin en l'écriture de l'altération traumatique de l'expérience subjective, c'est-à-dire aussi dans la manière dont l'écriture est altérée.

Par delà leurs différences au plan formel comme au plan des motifs de la narration et en particulier de la place explicite accordée à Auschwitz, tous les textes de Kertész convergent pour témoigner de l'expérience d'un sujet confronté à l'attaque de la possibilité même de raconter sa vie. Une attaque d'autant plus envahissante et destructrice que la vie est donnée pour invivable, la seule issue consistant à faire de sa vie « une réalité racontable » ${ }^{16}$. Toute l'œuvre de Kertész est tendue par cet effort : rendre sa vie racontable. Et cet effort de Kertész est aussi celui dominant la vie de tous les personnages principaux de ses textes, que leur statut soit clairement autoréférentiel 
ou que leur statut soit délibérément indécis, mixant le fictionnel et l'autoréférentiel.

C'est sans doute d'autant plus notable dans les textes caractérisés par l'indécision entretenue dans le jeu avec les identités de l'auteur, du narrateur et des personnages, particulièrement lorsqu'il y a une mise en abyme - comme dans Le refus, qui raconte le refus dans la Hongrie communiste d'un manuscrit écrit par un témoin survivant d'Auschwitz, manuscrit que le lecteur est amené à lire au travers de sa relecture par le personnage de l'auteur ; ou bien encore Roman policier, donné à lire comme la présentation par son avocat de la manière de confession écrite par un policier condamné pour avoir participé aux tortures organisées sous une dictature.

Non pas vivre pour raconter, mais plutôt raconter pour vivre, pour vivre la seule expérience vivable, à savoir l'incroyable de vivre, l'incroyable de l'existence. Et cet effort pour rendre sa vie vivable en racontant l'incroyable est montré par Kertész comme un effort continu à toute les périodes de sa vie, renvoyant à un étonnement d'être caractérisant la singularité d'une expérience intérieure continue de soi avant, pendant et après Auschwitz. A cet égard, il ne faut pas oublier que Kertész, né en 1929, a 14 ans quand il est déporté. De ce point de vue intérieur, malgré la souffrance et l'horreur, Auschwitz n'introduit pas une rupture, l'incroyable d'exister y préexiste. Et si Kertész va même jusqu'à parler dans Etre sans destin d'instants de «bonheur ${ }^{17}$ à Auschwitz, c'est probablement au sens où «dans les intervalles de la souffrance », cette expérience intérieure de soi dans l'incroyable de l'existence y trouve sa puissance maximale.

Si Auschwitz peut être considéré comme l'enclencheur de l'écriture, ce serait d'abord à ce titre-là, soit une expérience radicalisant la nécessité de parler sa vie à quelqu'un comme seule issue pour rendre vivable sa vie, toujours déjà par elle-même invivable, bien avant Auschwitz.

L'autre institué dans l'écriture de soi est seulement sollicité comme le témoin garant d'une vie racontable : celui auprès duquel on peut vivre sa vie en la racontant, celui sans la psyché duquel on ne peut se sentir exister, mais aussi celui avec lequel on défait sa vie en la racontant, dans la «collaboration » avec un processus continu d'anéantissement. Et cet autre contraint à collaborer à une telle collaboration prend la figure élective de la femme aimée et impossible à aimer.

\footnotetext{
Auschwitz, dis-je à ma femme, m'est apparu par la suite comme une exacerbation des vertus qu'on m'inculquait depuis ma prime jeunesse.
} 
Oui, c'est alors, durant mon enfance, durant mon éducation qu'a commencé mon impardonnable anéantissement, ma survie jamais survécue, dis-je à ma femme. J'ai pris une part modeste et pas toujours très efficace au complot silencieux ourdi contre ma vie, dis-je à ma femme. ${ }^{18}$

\section{Auschwitz au présent}

Cette certitude intérieure d'une «survie jamais survécue » depuis l'enfance, dont l'écriture vient témoigner, permet à Kertész de rendre sensible Auschwitz au présent, Auschwitz comme «révélateur de la vérité du siècle » (le $\mathrm{XX}^{\mathrm{ème}}$ ), comme réalité échappant à toute commémoration parce qu'elle ne peut se réduire à un événement qui se pourrait conjuguer au passé.

Avez-vous remarqué que dans ce siècle tout est devenu plus vrai, plus véritablement soi-même? Le soldat est devenu un tueur professionnel; la politique, du banditisme; le capital, une usine à détruire équipée de fours crématoires; la loi, la règle d'un jeu de dupes ; l'antisémitisme, Auschwitz; le sentiment national, le génocide. Notre époque est celle de la vérité, c'est indubitable. Et bien que par habitude on continue à mentir, tout le monde y voit clair; si l'on s'écrie : amour, alors tous savent que l'heure du crime a sonné, et si c'est : loi, c'est celle du vol, du pillage. ${ }^{19}$
La catastrophe d'Auschwitz a toujours lieu au présent, dans la mesure où le projet d'Auschwitz n'a pas été réfuté.

N'oublions pas qu'Auschwitz n'a pas été liquidé pour avoir été Auschwitz, mais parce que la fortune des armes a tourné ; et depuis Auschwitz, il ne s'est rien passé que nous aurions pu vivre comme la réfutation d'Auschwitz. En revanche, nous avons connu des empires fondés sur des idéologies qui se sont avérées dans la pratique n'être que de simples jeux de mots et c'est justement leur nature de jeu de mots qui les rendaient si utilisables, c'est-à-dire en faisait des instruments de terreur efficaces. ${ }^{20}$

Les camps staliniens ne sont pas les camps d'extermination nazis et le communisme n'est pas le nazisme. Mais il reste que le projet d'Auschwitz n'a pas été réfuté, à la fois au sens où il n'a pas été pleinement reconnu dans sa spécificité et où « le plus jamais ça »a été régulièrement démenti tant par la persistance de l'antisémitisme sous ses différentes formes que par la perpétration d'autres génocides. Surtout, la non réfutation d'Auschwitz se marque, au-delà même des effets traumatiques sur les survivants, sur les descendants des déportés et plus largement, sur l'ensemble des Juifs, dans le refus de prendre en compte les effets sur chaque être humain. 
Les humanistes professionnels voudraient croire qu'Auschwitz est arrivé uniquement à ceux auxquels il est arrivé précisément à cet endroit-là, en ce temps-là, mais que ceux auxquels il n'est pas arrivé précisément à cet endroit-là et en ce temps-là, c'est-à-dire la plupart des autres - l'Homme - eh bien, il ne leur est rien arrivé du tout. C'est-à-dire que l'éditeur aurait voulu lire dans mon roman que, malgré et justement malgré le fait que cela m'était arrivé à cet endroit-là et en ce temps-là, Auschwitz ne m'avait pas sali... il est à craindre que celui qui prend mon roman dans les mains avec de bonnes intentions et se met à le lire innocemment risque d'être quelque peu mêlé à cette saleté.

Je comprends donc très bien qu'un humaniste professionnel soit irrité par mon roman. Je suis moi-même aussi irrité contre les humanistes professionnels, parce qu'à travers leurs attentes, ils aspirent à mon anéantissement. ${ }^{21}$

La critique virulente de «l'innocence» de «l'humaniste professionnel » vise la négation d'Auschwitz dans sa forme la moins visible: elle dénonce la réception bien intentionnée du témoignage des survivants, supposés témoigner de leur victoire sur le mal et donc par là même témoigner d'une isolation du mal et en protéger tous les autres hommes, dispensés ainsi de s'y coltiner pour eux-mêmes dans la relation aux autres. Ce qui permet de sauver une image idéalisée de l'Homme. Qui n'a pas vécu Auschwitz ne saurait prétendre se réclamer d'Auschwitz pour vivre ou raconter sa vie, mais Auschwitz a révélé de façon catastrophique et irrémédiable les capacités autodestructrices de l'humanité au travers du potentiel destructeur et autodestructeur inscrit en chaque homme.

Chacun est ainsi entré avec Auschwitz dans «l'ère de la catastrophe ». Au plan collectif, il s'agit de l'entrée dans une période où «le diable » devient manifeste, «non pas dans le fait que l'homme tue, mais dans celui que les vertus indispensables au crime deviennent pour lui l'ordre du monde $»^{22}$. Au plan individuel, il s'agit de la marque de la figure du survivant apposée en chacun, inscrivant la menace d'une absence de destin, c'est-à-dire l'entrée du sujet dans l'absence de certitude intérieure concernant l'existence même de son moi, de l'entrée dans un univers de chaos: «sans retour possible vers un centre du Moi, vers une certitude inébranlable et indéniable du Moi : il est, au sens le plus propre du terme, perdu. L'être sans Moi, c'est la catastrophe, le Mal véritable $[\ldots] »^{23}$

\section{L'innocence catastrophique}

En d'autres termes, la présence d'Auschwitz se marque dans le primat d'un présent perpétuel, sans passé donc 
sans futur ${ }^{24}$, la dominance d'un monde où tout est formulable, où règne le mensonge sincère, soit le mensonge de soi à soi visant à préserver l'innocence d'une vie possible hors la catastrophe ${ }^{25}$. Deux emblèmes de cette innocence catastrophique, fondée sur l'ignorance de la catastrophe, sont particulièrement mis en valeur par Kertész : les touristes-fourmis et les « intellectuels moyens ». Les touristesfourmis mangent la réalité, surtout lorsqu'elle est en lien direct avec la catastrophe à ignorer: ils «emportent miette par miette mais sans relâche la signification des choses $»^{26}$.

Quant aux « intellectuels moyens », ils constituent les agents et supports électifs de la haine de la spiritualité. L'approche s'avère pleinement compatible et complémentaire par rapport à ce que Freud appelait la «haine de la culture », dans Malaise dans la culture. La « haine de l'esprit ${ }^{27}$ ou encore de $«$ haine de la gratitude ${ }^{28}$ désignent pour Kertész les principaux attributs de cette haine de la spiritualité, la spiritualité renvoyant au besoin de transcendance, mais dans le sens très précisément défini de l'ouverture d'une question : «Qui voit par nous ? » ${ }^{29}$

Voilà désignée la vérité du siècle révélée par Auschwitz: le besoin de transcendance comme objet de la haine des différentes versions idéologiques du « Tout est possible », du communisme au néo- libéralisme en passant par les intégrismes religieux - le «Tout est possible » n'étant pas séparable du «Tout est explicable » ou $\mathrm{du} \ll$ Tout est mesurable »

Cette attaque de l'énigme au cœur de la vie, de l'informulable au cœur de toute signification, qui constitue à ce titre une attaque du langage, Kertész entend en témoigner en tant que survivant, dans une écriture dépositaire de l'altération du sens de sa propre vie. Témoin survivant d'Auschwitz, Kertész se présente comme témoin de sa survie à un projet d'anéantissement qui préexiste à Auschwitz, dont Auschwitz n'est en somme que la confirmation et la radicalisation. L'écriture vient témoigner de «la survivance de la survie ${ }^{30}$ d'un homme qui n'a jamais cru à son existence $^{31}$, d'un homme inapte à trouver une cause ou un commencement à son impossibilité à répondre à la double question : «Qui suis-je ? Qui le sait ?» ${ }^{32}$.

\section{Ecriture et non-engendrement}

\section{Kaddish pour l'enfant qui ne naîtra} pas occupe ici une place à part, en proclamant une liaison entre écriture et engendrement ou plutôt nonengendrement. Il s'agit d'un récit autobiographique organisé autour du dialogue rétrospectif, après leur rupture, avec la femme aimée mais fuie pour son 
désir d'enfant. Le narrateur revendique l'écriture comme travail consistant à «creuser ma tombe ${ }^{33}$, un travail déjà entamé par d'autres. Mais il affirme en même temps la nécessité vitale de ce travail, décrit comme l'œuvre d'une « conscience morbide ${ }^{34}$ et exigeant d'être défendu contre la femme, au titre du domaine le plus sensible, le plus secret, le plus désarmé » de sa vie ${ }^{35}$. Le narrateur se décrit comme n'ayant pas d'autre choix pour vivre que «d'inexister ${ }^{36}$ dans l'écriture et d'assumer la cruauté de ce choix paradoxal d'une vie consacrée à «l'auto-liquidation publique ${ }^{37}$, en épuisant la femme confidente et son projet de le sauver d'une «survie jamais survécue ».

Le récit se fait ainsi le récit de l'enfermement progressif de la femme aimée dans une position bien particulière de témoin, il faudrait dire de témoin empêché d'être en relation avec lui jusqu'à être empêchée d'être: elle est prise à témoin de la complicité du narrateur avec son propre anéantissement présenté comme engagé dans l'enfance, notamment dans sa relation au père, et poursuivi avec Auschwitz $^{38}$. Radicalement, l'écriture comme besoin vital est garantie par la négation de l'engendrement: un non adressé à la venue de son enfant, à «mon existence considérée comme la possibilité de ton être $»^{39}$.
Pour permettre, on pourrait dire, de goûter avec bonheur la malédiction de la survie, c'est-à-dire d'une existence vécue comme impossible, l'écriture ne peut et ne doit vivre que fondée sur le meurtre de la possibilité de l'enfant. Kaddish pour l'enfant qui ne naîtra pas, en témoignant de l'épuisement mené à son terme de la relation à la femme aimée, s'adresse effectivement, par le biais du dialogue sans avenir avec elle, à l'enfant qui ne naîtra pas, pour lui déclarer «ton inexistence considérée comme la liquidation radicale et nécessaire de mon existence $\gg{ }^{40}$. Mais cette inexistence de l'enfant, il faut bien voir qu'elle est partagée par l'auteur-narrateur, que l'écriture est en somme leur lieu commun ou, pour le dire autrement, que l'écriture permet de vivre l'inexistence. Vivre l'inexistence, c'est-à-dire l'inexistence de l'enfant pas né d'Imre Kertész, mais aussi le sentiment d'inexister de l'enfant mal né nommé Imre Kertész, enfant resté dans les limbes, enfant mal conçu et survivant à sa naissance, enfant dont la précarité du moi témoigne d'une absence de projet, ou, pour le dire avec les mots de Kertész lui-même, d'une absence de destin.

Si Kertész écrit pour se libérer du futur $^{41}$, si l'écriture se veut l'affirmation sans cesse réitérée qu'il n'y avait pas d'autre vie possible que celle qu'elle décrit, qui n'a précisément d'existence 
qu'écrite, si cette vie ne peut être écrite qu'en traçant une continuité de l'enfance jusqu'à Auschwitz et ensuite la vie d'un écrivain marginalisé dans la Hongrie communiste puis post-communiste, c'est qu'il est venu dans un monde qui n'était pas prêt pour sa venue, un monde caractérisé par la menace d'une double catastrophe, l'arrivée au pouvoir d'Hitler et la séparation de ses parents.

[...] Quand je suis venu au monde, le soleil était dans le signe de la plus grande crise économique jamais connue...les hommes se jetaient à l'eau, sur le pavé, dans le vide, selon les circonstances; un chef de parti du nom d'Adolf Hitler m'a montré un visage extrêmement hostile d'entre les pages de son livre intitulé Mein Kampf, la première loi hongroise antijuive, appelée numerus clausus, était au zénith de ma constellation, avant d'être remplacée par les suivantes [...] Tous les signes de la terre $[\ldots]$ témoignaient $\mathrm{du}$ caractère superflu voire déraisonnable de ma naissance. De surcroît, j'étais arrivé comme un fardeau pour mes parents : ils s'apprêtaient à divorcer. Je suis la matérialisation de l'amour d'un couple qui ne s'aimait plus $[\ldots]^{42}$

\section{La menace}

Issu d'une séparation catastrophique dans un monde voué à la catastrophe, donc seul et abandonné dès la naissance, il ne peut pas se voir, il ne peut pas se voir dans les causes présidant à sa venue. A cet égard, le roman intitulé Liquidation montre, sans doute sur un mode cathartique, ce qui a peut-être constitué une menace pour l'écrivain Kertész : le suicide non seulement comme accomplissement de l'inexistence au sens d'une absence de destin, mais comme moyen d'autodétruire son pouvoir d'engendrement, tant au plan de la conception d'une œuvre littéraire qu'au plan de la conception d'un enfant. Le roman montre la réussite de ce projet d'autodestruction au travers du personnage principal, qui est, comme Kertész, écrivain, traducteur, survivant d'Auschwitz. Ce personnage, décrit comme cherchant à vivre Auschwitz au présent, à Budapest, dans tous les registres de sa vie, sans $\operatorname{limites}^{43}$, se fabrique un destin en se suicidant, après avoir organisé la destruction de son œuvre en utilisant la femme aimée. «Il déteste le nom qu'il a reçu de ses ancêtres, tout comme il déteste ses ancêtres et tous ceux qui sont la cause de son existence $»^{44}$.

Ce n'est certes pas un hasard que ce soit précisément dans ce roman que figure l'histoire d'un enfant né à Auschwitz et sauvé dans des circonstances exceptionnelles, et que cette histoire soit jugée par le personnage de l'écrivain suicidaire comme une anecdote qui en tant 
que telle, bien qu'elle soit réelle, ne peut prétendre à la vérité.

Il m'expliqua que le problème résidait justement là. Cela s'était passé et pourtant ce n'était pas vrai. C'était une exception. Une anecdote. Un grain de sable tombé dans le mécanisme à broyer les cadavres. ${ }^{45}$

En fait, cet enfant né à Auschwitz et dont l'existence ne peut pas véritablement prendre sens, c'est vraisemblablement Kertész pour Kertész, un Kertész qui ne peut radicalement pas envisager d'être père, tel que c'était exprimé dans Kaddish: «Non je ne pourrai jamais être le père, le destin, le dieu d'un autre être. Non jamais ne peut arriver à un autre enfant ce qui m'est arrivé dans mon enfance. $»^{46}$

En 2003, date de publication de Liquidation, l'écriture fictionnelle peut fonctionner pour Kertész comme catharsis, quarante ans après Auschwitz, l'essentiel de son œuvre déjà écrit et étant assuré d'être reconnu à la fois comme écrivain et comme témoin survivant, un an après la consécration du Nobel. En 1988, la situation est différente, lorsqu'il publie $L e$ Refus, à mi-chemin du roman autobiographique et de l'autofiction. Le récit est centré sur le personnage principal, un écrivain et traducteur désigné comme «le vieux », qui raconte des années après le refus par les éditions de l'Etat de son récit de déportation à Auschwitz, tout en le relisant. Ce récit contient en abyme plusieurs autres récits. L'autocommentaire, présent à la fois dans le récit en train d'être relu et dans le récit de la relecture, consiste surtout pour «le vieux» à lire ce texte comme le seul vrai texte qu'il ait écrit, le seul qui ne consistait pas en une commande, son seul livre d'écrivain, celui avant et après lequel il ne peut exister dans l'écriture, se contentant d'exister grâce à l'écriture comme (maigre) gagne-pain et de rêver de «devenir un écrivain qui n'aurait plus besoin d'écrire ${ }^{47}$ pour gagner sa vie.

Avec la mise en abyme d'un personnage qui ne parvient pas à écrire un récit autobiographique et finit par en mourir, Le refus met en exergue la tentation dangereuse de l'écriture de soi, lorsqu'elle promet de se venger contre le monde qui a rendu possible son destin malheureux, en proclamant qu'il n'y a pas d'autre possibilité pour sa vie que ce $\operatorname{destin}^{48}$. Une telle proclamation met en danger l'écriture de soi tout autant que l'écrivain, par ce qu'elle se fonde sur la croyance d'un moi plénier. C'est pourquoi Kertész ne cesse de ruser avec l'écriture de soi, à laquelle il refuse le credo autobiographique d'un moi plénier, en la diffractant, en démultipliant les points de vue narratifs, en s'appuyant ou non sur un mixage fictionnel. Cela lui permet à la fois de proclamer qu'il n'y a pas d'autre 
possibilité à vivre que la vie en train d'être vécue, tout en récusant l'idée de destin: ainsi le refus du texte raconté dans Le refus est-il présenté comme relevant non pas du destin mais «de l'effondrement d'un illusion : moi-même ${ }^{49}$. L'illusion du moi plénier, c'est l'illusion de la «vie formulable ».

«Pourquoi moi ?», se demande dans un rêve le personnage du récit dans le récit, lorsqu'il se voit seul pour sauver un homme en train de se noyer ${ }^{50}$. Il pourrait s'agir ainsi de figurer avec le noyé « sa vie délaissée », une vie déjà vécue mais dont il reste à sauver le récit ${ }^{51}$. Ecrire, lorsqu'il s'agit de témoigner d'une expérience de soi, passe par un combat à la fois avec et contre les souvenirs : sauver ses souvenirs, mais aussi se sauver de ses souvenirs, de la réduction à ses souvenirs, grâce à l'écriture.

Le but n'est pas d'expliquer sa vie mais de la rendre vivable en la racontant, sans tomber dans le piège de vouloir réunir des preuves ou de constituer soi-même une preuve pour les autres ${ }^{52}$. Ce piège menace tout témoin survivant, confronté à ceux qui sollicitent son témoignage sans assumer leur ambivalence: dis-moi comment c'était, c'est-à-dire donne moi des preuves de ce que tu as vécu. Une demande de preuves mettant d'autant plus le survivant aux prises avec un désaveu de son expérience traumatique, qu'il ne peut se dérober.
Face à un tel piège, la juste position de l'écriture de soi lorsqu'elle assume de témoigner serait de devenir une preuve pour soi-même. Il reste que cela dépend des autres !

\section{La confiance malgré tout}

L'absence de sens semble régner sur la vie telle qu'elle est présentée par l'écriture de soi, dans cette convergence malheureuse d'une naissance venant annoncer paradoxalement la séparation des parents et de la catastrophe nazie, dans cette continuité entre la défaillance de l'accueil parental et celle du monde tel qu'il va être organisé par les nazis. Mais avec son dernier texte, Dossier $K$, la perspective change, ou plutôt, une perspective est introduite avec la notion de confiance.

Dossier $K$. constitue le seul texte de Kertesz à être explicitement revendiqué comme une autobiographie, même s'il s'agit de la réécriture d'entretiens menés en 2003-2004 avec son éditeur hongrois. Le texte se présente sous la forme d'un dialogue cette fois-ci avec un interlocuteur dégagé de toute dimension fictionnelle, contrairement aux autres textes de Kertesz, à l'exception d'Etre sans destin. Le dialogue n'est plus un dispositif fictionnel permettant de mettre en scène le dialogue intérieur de l'auteur, mais un mode 
d'écriture de soi référé aux dialogues de Platon et faisant officiellement place à l'autre en position non seulement d'interlocuteur, mais de témoin garant d'une écoute, c'est-à-dire d'une approche relationnelle de soi, hors la solitude du soliloque interne, fût-il construit pour être donné à lire aux autres. Il y a donc une confiance affichée dans les mots pour se dire, ou du moins pour se donner à écouter, en renonçant à la protection romanesque. Et le thème de la confiance parcourt d'ailleurs tout le texte, mais essentiellement en référence à la figure suicidée de Jean Amery, qui selon Kertesz est celui qui a le mieux pensé «le sentiment de détresse et de désespoir absolu » ${ }^{53}$ vécu par les déportés dans les camps nazis.

[...] c'est peut-être Jean Amery qui nous en apprend le plus dans ses essais. Il a un mot exceptionnellement précis pour cela : Weltvertrauern, que je traduirais par: la confiance accordée dans le monde. Il décrit à quel point il est difficile de vivre ans cette confiance. Une fois qu'on l'a perdue, on est condamné à vivre éternellement seul parmi les hommes. On ne voit plus jamais en autrui son prochain, mais son ennemi. (respectivement Mitmenschen et Gegenmenschen, dans l'original [Mitmenschen: humain avec, Gegenmenschen: humain contre]. Luimême a perdu cette confiance sous les coups de la Gestapo qui l'a torturé [...]

\begin{abstract}
Bien qu'il ait survécu au camp de concentration d'Auschwitz, il a lui-même exécuté la sentence plusieurs décennies après : il s'est suicidé. ${ }^{54}$
\end{abstract}

La confiance est donc abordée d'abord négativement, comme l'objet même de la destruction nazie, en deçà du génocide lui-même: une destruction visant, à la fois à l'intérieur de chacun et dans la relation aux autres, la subjectivité, le sentiment d'exister dans un lien indéfectible à l'autre comme semblable.

Il est significatif que pour illustrer positivement la confiance dans le monde, Kertesz mentionne sa mère, qu'il décrit comme dotée d'un égoïsme de vie suffisamment fort pour nier le danger et aller demander des nouvelles de son fils déporté à un officier nazi, avec son étoile jaune $^{55}$. Et il souligne la dimension infantile de cette confiance, en nous donnant à penser qu'il en a hérité en quelque façon, suffisamment pour survivre à Auschwitz, du fait de son âge, ce qui n'aurait sans doute pas été possible à ses yeux s'il avait été déporté à l'âge adulte.

[...] il n'est pas facile d'enlever sa joie de vivre à un enfant de quatorze ans, surtout s'il est entouré de camarades de son âge avec lesquels il peut partager son destin. Il a en lui une espèce... une espèce de naïveté innocente qui le protège du sentiment de détresse et de désespoir absolu. En ce sens, il est plus facile de 
briser un adulte. [...] dans l'état de délabrement moral où j'étais, cette confiance... n'était sans doute pas radieuse, mais elle était certainement visible. Je m'imaginais tout simplement que le devoir du monde des adultes était de me tirer de là, de me ramener sain et sauf à la maison. Tout cela paraît assez ridicule aujourd'hui, mais c'est vraiment ce que je ressentais. Et je crois dur comme fer que j'ai été sauvé par cette confiance infantile. $^{56}$

\section{Confiance et au-delà}

Il reste que pour Kertesz, il y a un au-delà de la mère, mais ignoré d'ellemême, et d'autant plus difficilement transmissible que le père fait défaut et qu'il est expressément visé par la destructivité nazie. La confiance aveugle de la mère est utile pour la survie et l'adaptation à un monde meurtrier, mais insuffisante pour vivre une vie d'homme, donner sens à la vie. Toutefois, il y a pour Kertesz un audelà des parents qui donnent la vie et qui, donnant du même coup la mort ${ }^{57}$, doivent aider l'enfant, par leurs attentions psychiques, à transformer sa haine à leur égard en force de vie, ce dont l'enfant Kertesz n'a manifestement pas bénéficié. Malgré tout, l'écriture de soi dans Dossier $K$ parvient à témoigner de cet au-delà : «On doit des remerciements à quelqu'un pour notre vie, même s'il se trouve qu'il n'y a personne pour les recevoir $»^{58}$. Il y a pour Kertesz, dans cette gratitude inconditionnelle, un au-delà de toute explication, qu'il s'agisse du don de la vie ou qu'il s'agisse de son envers absolu, la Shoah $^{59}$. La possibilité d'être est intrinsèquement liée à cet au-delà, qui est pour lui expérimentable dans l'écriture de soi, dans le fait même d'écrire et de donner à lire sa vie à l'autre, abstraction faite de la vie elle-même réduite dans sa dimension traumatique à «une survie jamais survécue ».

Raconter sa vie pour la vivre, rendre sa vie malgré tout vivable en la racontant à un autre institué dans l'écriture de soi comme témoin garant d'une écoute revient en somme à matérialiser l'au-delà au titre d'un besoin de transcendance: «Qui voit par nous?» ${ }^{60}$ Kertész, dont toute l'œuvre peut se lire comme une méditation autour de la notion de destin, propose ainsi une approche de la spiritualité dans les termes d'un point de vue sans lequel une vie ne peut se regarder, d'un regard sur la vie à la fois nécessaire à la vie et n'existant qu'au travers de chaque vie.

L'écriture de soi, en se confiant à un autre garant d'une écoute, matérialise l'au-delà comme le point à partir duquel on peut se voir, c'est-à-dire se voir regardé par l'autre grâce à la confiance accordée aux mots. L'écriture de soi sauve de l'anéantissement de la confiance engagé 
par la double catastrophe intime et collective, et sauve le futur, un futur qui ne peut être consacré par l'engendrement mais par la création.

Ecrivant sa vie pour quelqu'un, on la donne à voir par les mots et leur adresse. La destination des mots dessine un regard interprétant la vie, inscrivant le sens de la vie.

\section{Notas}

${ }^{1}$ Psychologue clinicien et psychanalyste, professeur de psychopathologie à l'Université Paris 13, UTRPP EA 3413.

${ }^{2}$ En deçà même des limites naturelles de la psychanalyse, celles liées à son champ d'investigation comme celles liées à sa nature transférentielle.

3 Cf. notamment Lejeune P., Le pacte autobiographique, Paris, Le Seuil, 1975.

${ }^{4}$ Cf. notamment Chiantaretto J.-F., De l'acte autobiographique. Le psychanalyste et l'écriture autobiographique, Paris, Champ Vallon, 1995.

${ }^{5}$ Cf. notamment Chiantaretto J-F., Clancier

A., Roche A. (dir.), Autobiographie, journal intime et psychanalyse, Paris, Anthropos/Economica, 2005.

${ }^{6}$ Dans l'ensemble du livre, le Je sera défini en référence à Piera Aulagnier: le sujet parlant (cf.Aulagnier P., La violence de l'interprétation, coll. «Le fil rouge», Paris, PUF, 1975).
${ }^{7}$ Le nourrisson et plus largement, le petit d'homme avant l'entrée dans le langage, avant l'accès au je - le je au sens grammatical, qui conditionne le Je marquant l'accès au statut de sujet parlant (étant entendu que le second ne se réduit pas pour autant au premier).

8 Grenier L., Les violences de l'autre, Montréal, Québécor, 2008, p. 160.

${ }^{9}$ Je reprends ici ma formulation de 2005 (Chiantaretto J.-F., Le témoin interne. Trouver en soi la force de résister, Paris, Aubier, 2005).

${ }^{10}$ C'est la lecture de Primo Levi qui m'a amené à proposer le concept de «témoin interne ».

${ }^{11}$ Cf. par ex. Levi P. (2002), L'asymétrie ou la vie, Paris, 10/18, 2005, p. 187.

12 Je rappelle brièvement les principaux textes (traduits en français) d'Imre Kertész, prix Nobel de littérature en 2002, né dans une famille juive de Budapest en 1929, déporté à Auschwitz puis à Buchenwald. Tous ses textes mêlent l'écriture de soi et des aspects fictionnels plus ou moins développés et tous abordent Auschwitz, même si dans certains cas ce n'est qu'allusivement. Il n'empêche qu'il faut distinguer Etre sans destin, publié en 1975, récit testimonial explicitement centré sur Auschwitz, racontant le parcours de vie d'Imre Kertész de l'enfance jusqu'à son retour de déportation. On pourrait aussi essayer de distinguer malgré tout deux 
types de textes, les textes au statut clairement autoréférentiel et ceux dont le statut est délibérément indécis, mixant le fictionnel et l'autoréférentiel, le roman ou la nouvelle et l'écriture de soi. Dans la première catégorie, figureraient trois textes formant un triptyque: Etre sans destin (1975), Kaddish pour l'enfant qui ne naîtra pas (1990), Un autre (1997). Et dans la seconde: Roman policier (1977), Le refus (1988), Liquidation (2003) et des nouvelles comme Le drapeau anglais (1991), Procès verbal (1991) ou Le chercheur de traces (1998). Le seul texte relevant strictement de l'écriture autobiographique est Dossier K (2006).

${ }^{13}$ Kertész I. (1997), Un autre, Arles, Actes Sud, 1999, p. 114.

14 Kertész I. (1975), Être sans destin, Arles, Actes Sud, 1998, p. 361.

${ }^{15}$ Ibid., p. 165.

${ }^{16}$ Cf. la citation plus haut: Kertész I. (1997), op. cit., p. 114.

${ }^{17} \mathrm{Cf}$. la citation plus haut : ibid., p. 361.

${ }^{18}$ Kertész I. (1990), Kaddish pour l'enfant qui ne naîtra pas, Arles, Actes Sud, 1995, pp. 146-147.

${ }^{19}$ Kertész I. (1997), op. cit., pp. 84-85.

${ }^{20}$ Ibid., p. 84-85.

${ }^{21}$ Kertész I. (1988), Le refus, Arles, Actes Sud, 2001, p. 40.

${ }^{22}$ Ibid., p. 51.

23 Kertész I. (2003), Liquidation, Arles, Actes Sud, 2004, p. 59.
${ }^{24}$ Cf. Ibid., p. 125.

25 Cf. Kertész I. (1991), Le drapeau anglais, Arles, Actes Sud, 2005, pp. 31-32. ${ }^{26}$ Kertész I. (1998), Le chercheur de traces, in Le drapeau anglais, Arles, Actes Sud, 2005, p. 125.

${ }^{27}$ Kertész I. (1997), op. cit., p. 86.

${ }^{28}$ Ibid., p. 93.

${ }^{29}$ Ibid., p. 86.

${ }^{30}$ Kertész I. (1990), op. cit., p. 79.

${ }^{31}$ Kertész I. (1988), op. cit., p. 32.

${ }^{32}$ Kertész I. (1990), op. cit., p. 156.

${ }^{33}$ Kertész I. (1990), op. cit., p. 112

${ }^{34}$ Ibid., p. 153.

${ }^{35}$ Ibid., p. 110.

${ }^{36}$ Kertész I. (1988), op. cit., p. 76.

${ }^{37}$ Kertész I. (1997), op. cit., pp. 66-67.

${ }^{38}$ Id., p. 146.

${ }^{39}$ Ibid., p. 10.

${ }^{40}$ Ibid., p. 44.

${ }^{41}$ Kertész I. (1988), op. cit., p. 43.

${ }^{42}$ Ibid., pp. 87-88.

${ }^{43}$ Kertész I. (2003), op. cit., p. 77.

${ }^{44}$ Ibid., p. 36.

${ }^{45}$ Ibid., p. 37-38.

${ }^{46}$ Kertész I. (1990), op. cit., p. 119.

${ }^{47}$ Ibid. pp. 321-322.

${ }^{48}$ Ibid., pp. 289-90.

${ }^{49}$ Ibid., p. 69.

${ }^{50}$ Kertész I. (1988), op. cit., p. 18.

${ }^{51}$ Ibid., pp. 345-46.

${ }^{52}$ Cf. notamment Kertész I. (1998), op. cit., p. 132. 
53 Kertész I. (2006), Dossier K, Arles, Actes Sud, 2008, p. 19.

${ }^{54}$ Ibid., p. 19.

${ }^{55}$ Ibid., p. 21.

${ }^{56}$ Ibid. pp. 19-20.

${ }^{57}$ Ibid., p. 58.

${ }^{58}$ Ibid., p. 36.

${ }^{59}$ Ibid., p. 72.

${ }^{60}$ Ibid., p. 86.

\section{Bibliographie}

Aulagnier P. (1975). La violence de l'interprétation, coll. «Le fil rouge», Paris, PUF, 1975.

Chiantaretto J.-F. (1995). De l'acte autobiographique. Le psychanalyste et l'écriture autobiographique, Paris, Champ Vallon.

Chiantaretto J.-F. (2005). Le témoin interne. Trouver en soi la force de résister, Paris, Aubier.

Chiantaretto J-F., Clancier A., \& Roche A. (dir.). (2005). Autobiographie, journal intime et psychanalyse, Paris, Anthropos/Economica.

Grenier L. (2008). Les violences de l'autre, Montréal, Québécor.

Kertész I. (1975), Être sans destin, Arles, Actes Sud, 1998.

Kertész I. (1988), Le refus, Arles, Actes Sud, 2001.

Kertész I. (1990), Kaddish pour l'enfant qui ne naîtra pas, Arles, Actes Sud, 1995.
Kertész I. (1991), Le drapeau anglais, Arles, Actes Sud, 2005.

Kertész I. (1997), Un autre, Arles, Actes Sud, 1999.

Kertész I. (1998), Le chercheur de traces, in Le drapeau anglais, Arles, Actes Sud, 2005.

Kertész I. (2003), Liquidation, Arles, Actes Sud, 2004.

Kertész I. (2006), Dossier K, Arles, Actes Sud, 2008.

Lejeune P. (1975). Le pacte autobiographique, Paris, Le Seuil.

Levi P. (2002), L'asymétrie ou la vie, Paris, 10/18, 2005.

Jean-François Chiantaretto - Psicólogo clínico e psicanalista; atuou em vários centros de saúde, incluindo o Alfred Binet Centre, Paris. É doutor em Filosofia e professor de Psicopatologia na Universidade Paris 13. Fundou e organiza desde 1992 o grupo de pesquisa Littérature personnelle et psychanalyse. É diretor da Unité Transversale de Recherche em Psychogenèse et Psychopathologie na Universidade de Paris 13. Tem publicado diversos livros sobre Psicanálise, linguagem, escrita.

E-mail: jfchant@wanadoo.fr 